While B (and her husband) are not concerned about future readmission to a mixed ward, would a single sex ward be a more appropriate environment irrespective of her expressed wishes? In practice an extra contractual referral would have to be made to achieve this care.

RICHARD HODGSON, The Sutherland Centre, Belgrave Road, Dresden, Staffordshire ST3 4PN

\section{Unerpected therapeutic benefit of Community Care Order application}

Sir: I wish to report an unexpected therapeutic benefit of an application for a Community Care Order (CCO). It is not known if the benefit was attributable to the specific features of this new legislation or simply therapeutic attention. The application was made as follows:

Day 1 -Mental Health Officer (MHO) telephoned the patient at home to introduce himself and arrange a home visit. Day 2-MHO visited and introduced himself as the After-Care Officer (ACO). Responsible Medical Officer (RMO) telephoned later to arrange a visit. Day 3-RMO visited and introduced himself as the Special Medical Officer (SMO). Both completed a Medical Recommendation. The General Practitioner (GP) telephoned after lunch to introduce himself. GP visited and completed Medical Recommendation.
RMO (not SMO) tried to telephone that evening to obtain further information about the patient's nearest relative whom she last saw in 1975 . Telephone engaged; MHO and ACO had telephoned again. Days 4 and 5-MHO and ACO visited and the latter completed After-Care Report. Day 6 - ACO did not visit the patient, but the RMO and SMO instead. Day 7 (Sunday) People with acronyms had a day off. Day 8 Patient shopped in the morning and went to the laundrette in the afternoon, saying that she would not be at home to visitors. Day 9-As required the RMO delivered the $C C O$ application to the patient before it was lodged with the Sheriff Clerk. RMO told subsequently that he was not competent to tell the patient about the date of the Hearing. A Sheriff Officer was to visit the next morning to notify the patient.

Day 10 - Sheriff Officer did not visit or notify the patient. As required the RMO visited in the afternoon "to encourage" the patient to attend a hearing about which she knew nothing.

Whereas the patient used to be sullen and socially isolated, she was cheerful and no longer socially isolated by the time the application was lodged; she even bought an extra packet of biscuits for her visitors.

Allan I. F. ScOTT, Andrew Duncan Clinic, Royal Edinburgh Hospital, Edinburgh, EH10 5HF 